\title{
Kernos
}

Revue internationale et pluridisciplinaire de religion grecque antique

$25 \mid 2012$

Varia

\section{Le rite sacrificiel antique : la longue durée et la fin du sacrifice}

Christoph Auffarth

\section{(2) OpenEdition \\ Journals}

Édition électronique

URL : http://journals.openedition.org/kernos/2029

DOI : 10.4000/kernos.2029

ISSN : 2034-7871

Éditeur

Centre international d'étude de la religion grecque antique

Édition imprimée

Date de publication : 26 octobre 2012

Pagination : 297-303

ISSN : 0776-3824

Référence électronique

Christoph Auffarth, « Le rite sacrificiel antique : la longue durée et la fin du sacrifice », Kernos [En ligne], 25 | 2012, mis en ligne le 01 octobre 2012, consulté le 21 septembre 2020. URL : http:// journals.openedition.org/kernos/2029; DOI : https://doi.org/10.4000/kernos.2029 
Kernos 25 (2012), p. 297-395.

\title{
Revue des Livres
}

\author{
1. Articles critiques
}

\section{Le rite sacrificiel antique : la longue durée et la fin du sacrifice \\ par Christoph Auffarth}

\section{Le rite sacrificiel comme « origine sauvage » de la religion}

Le sacrifice comme élément central des religions antiques a fasciné la science des religions depuis que celle-ci pense avoir compris le rituel comme le véritable «langage » de la religion ${ }^{1}$. La découverte du rituel a apporté simultanément deux modèles d'interprétation dans le discours sur la religion : la religion est, dans l'homme, quelque chose d'archaïque, de primitif, de non apprivoisé par la culture. Par conséquent, la religion n'est pas ce que les théologiens veulent entendre par là aujourd'hui : un comportement éthique et son fondement ultime dans le transcendant, le respect pour l'infini, la sublimation suprême, en somme! À l'origine, la religion s'avère être un comportement préconscient, qui se trouve en relation avec les instincts de l'homme en tant qu'être naturel2 ${ }^{2}$. Une « situation primitive» en est le parfait exemple ${ }^{3}$.

Un ermite chrétien, Nil, raconte, au IV s. ap. J.-C., une rencontre émotionnellement tendue. En situation d'extrême besoin, des nomades arabes se saisissent du chameau sur

* Le point de départ de cette contribution est la lecture de l'ouvrage de Maria-Zoe PeTropoulou, Animal sacrifice in ancient Greek religion, Judaism, and Christianity 100 BC - AD 200, Oxford, UP, 2008 (Oxford Classical Monographs). Je remercie Vinciane Pirenne-Delforge pour son soutien dans la transformation de ce compte rendu en une réflexion plus ample, Tilman Hannemann pour son assistance dans la rédaction et tout particulièrement Aurian Delli Pizzi pour la traduction de ma contribution. - Je la dédie à Jörg Rüpke pour son cinquantième anniversaire.

1 Jan Bremmer, «'Religion', 'Ritual' and the Opposition 'Sacred vs. Profane': Notes Towards a Terminological 'Genealogy'», in Fritz GRAF (éd.), Ansichten griechischer Rituale. Festschrift für Walter Burkert, Stuttgart/ Leipzig, Teubner, 1998, p. 9-32. Plus largement, Jan BrEMMER, «Myth and Ritual in Ancient Greece: Observations on a Difficult Relationship ", in Raban VON HAEHLING (éd.), Griechische Mythologie und Frübchristentum, Darmstadt, WBG, 2005, p. 21-43.

2 Sigmund Freud, Totem und Tabu. Einige Übereinstimmungen im Seelenleben der Wilden und der Neurotiker, 1912/1913, Ausgabe S.F., Studienausgabe, Vol. 9, Frankfurt a. M., S. Fischer, 1974, p. 287-444, ici p. 418 :


anderes, als was spätere Zeiten darunter verstanden: die Darbringung an die Gottheit, um sie zu versöhnen oder sich geneigt zu machen. (Von dem Nebensinn der Selbstentäußerung ging dann die profane Verwendung des Wortes aus. ... Das Opfer war nachweisbar zuerst nichts anderes als "an act of social fellowship between the deity and his worshippers" [SMITH, Semites, 18942 , 224], ein Akt der Geselligkeit, eine Kommunion der Gläubigen mit ihrem Gotte ». Toute la section des pages 417-424 est fondée sur Smith.

${ }^{3}$ Freud, Tabu, 1913, p. 418 : « Das Tieropfer ist älter als der Ackerbau. » Viande et sang sont mangés «älter als der Gebrauch des Feuers ». 
le Sinai, pour apaiser leur faim ${ }^{1}$. Ils le lacèrent à mains nues, se gavent avidement de la chair crue. C'est un animal qui leur est sacré, c'est leur totem qu'ils «mettent à mort »². Personne n'est coupable individuellement, ils agissent ensemble et simultanément. La transgression commune du tabou soude le groupe.

Ce récit exotique devient un texte de référence pour le sacrifice dans sa forme originelle. Les Grecs se seraient également considérés, par rapport au sacrifice, comme des ßouৎóvot ${ }^{3}$, des «tueurs de bœufs »4. William Robertson Smith compare la communion chrétienne à ses contemporains écossais sur ce point : ils mangent, eux aussi, leur totem, le Christ ${ }^{5}$. Ce qui, pour les Arabes et les Sémites, apparait encore comme une rupture orientale devient par la suite universellement humain. Sigmund Freud reconnait des correspondances entre le rituel religieux (de la religion primitive comme celle de ses contemporains) et le trouble obsessionnel compulsif des névrosés. Il peut donc considérer qu’il faut rejeter le principe de la religion comme étant une forme de maladie mentale 6 . De modèle classique, l'antiquité devient le contre-exemple sauvage et sombre. Friedrich Nietzsche, Jane Harrison, Martin Nilsson renversent l'image de la « noble simplicité et calme grandeur $»^{7}$. L'« origine sauvage $»^{8}$ du rituel devient, en somme, la clef de la religion; l'homme devient l'homo necans ${ }^{9}$. Dès lors nait une théorie de la culture qui attribue au sacrifice, en tant que rituel, un effet canalisateur : grâce au rite sacrificiel, la menaçante agressivité intraspécifique est déchargée sur l'animal. Au lieu de s'entretuer, les hommes mettent à mort l'animal sacrificiel. Walter Burkert et René Girard, le spécialiste de la littérature, développent simultanément une théorie semblable sur le rite sacrificiel, du point de vue de la culpabilité, de l'imitation, de l'évacuation de l'agressivitée ${ }^{0}$. Plus large et plus systématique est l'interprétation du sacrifice comme ordre et représentation du groupe $^{11}$.

\footnotetext{
1 [Nilus], narratio 3 [Migne, PG 79, 612B-614C]. Traduction anglaise: Daniel F. CANER, History and bagiography from the late Antique Sinai, including translations of Pseudo-Nilus' Narrations, Ammonius' Report on the slaughter of the monks of Sinai and Rhaithou, and Anastasius of Sinai's Tales of the Sinai Fathers, Liverpool, UP, 2010, p. 73-105. Michael LINK, Die Eræählung des Pseudo-Neilos. Ein spätantiker Märtyrerroman. Einleitung, Text, Übersetzung, Kommentar, Munich, Saur, 2005 (Beiträge zur Altertumskunde, 220).

2 W. Robertson Smith, Die Religion der Semiten. Traduction allemande d'après la seconde édition des Lectures on the religion of the Semites de R. Stübe, Fribourg s. B., Mohr, 1899, p. 162-182, 262 sq. Tabu (sur les animaux sacrés) utilise Smith p. 187-189; Totem comme "dieux» p. 99-101; la consommation du totem comme violation d'un tabou p. 217, 226 sq.

${ }^{3}$ Freud, Tabu, 1913, p. 422 cite sur ce point SMITH, Semites, 1894², p. 304.

${ }^{4}$ Corroboré par Walter Burkert avec tout son savoir-faire en études grecques dans Walter BURKERT, Homo necans. Interpretationen altgriechischer Opferriten und Mythen, Berlin, de Gruyter, 1972 (RGVV, 32), p. 154161. Reproduction avec postface $1997^{2}$. Traduction américaine : Harvard, 1983. Traduction française : Paris, 2005. Albert Henrichs atténue la composante - ழóvoৎ: pas de meurtre! «Gott, Mensch, Tier: Antike Daseinsstruktur und religiöses Verhalten im Denken Karl Meulis », in Fritz GrAF (éd.), Klassische Antike und neue Wege der Kulturwissenschaften. Symposium Karl Meuli, Bâle, 1992 (Beiträge zur Volkskunde, 11), p. 129-167.

${ }^{5}$ Sur ce point, voir Bernhard MAIER, William Robertson Smith. His life, his work and his times, Tübingen, Mohr Siebeck, 2009 (FAT, 67), p. 258-269. Marjorie WHEELER-BARCLAY, The science of religion in Britain, 18601915, Charlottesville, Virginie, UP, 2010.

${ }^{6}$ Freud, Tabu, 1913, p. 430; 438.

${ }^{7}$ Sur le contexte, voir Hubert CANCIK, Nietzsches Antike, Stuttgart/Weimar, 1995.

${ }^{8}$ C'est ainsi ("Wilder Ursprung») que Glen MOST a nommé le Sammlung kleiner Schriften de Walter Burkert, Berlin, 1990, édité par ses soins.

${ }^{9}$ BURKERT, Homo necans.

${ }^{10}$ René GIRARD, La Violence et le sacré, 1972 [all. Das Heilige und die Gewalt, Frankfurt a. M., Fischer, 1994. Düsseldorf, Patmos, 2006]. Des choses cachées depuis la fondation du monde, 1978 [all. Das Ende der Gewalt. Analyse des Menschheitsverbängnisses, Herder, Fribourg, 2009].

${ }^{11}$ Burkhard GLAdigow, « Die Teilung des Opfers. Zur Interpretation von Opfern in vor- und frühgeschichtlichen Epochen », Frübmittelalterliche Studien 18 (1984), p. 19-43.
} 


\section{Longue durée, origine et développement}

À l'étude de l'origine du sacrifice (comme acte central de la religion) pour chaque culture est associée une théorie de la culture qui ne fait pas réellement apparaittre «l'homme primitif dans la culture tardive » comme adapté au monde moderne'. Ainsi l'intérêt de l'équipement génétique de l'homme sauvage a pris sa valeur en relation avec les primates et d'autres créatures grégaires dans le cadre de la biologie théorique ${ }^{2}$. Que sont les dix mille ans depuis la première cité, que sont ces quelques dix mille ans depuis l'apparition de l'agriculture et de la sédentarisation en comparaison des centaines de milliers d'années d'existence de l'homme comme chasseur? Mais quelle valeur peut-on accorder à la thèse de la continuité de l'bomo necans du chasseur au sacrificateur ?3 Et y a-t-il réellement un lien avec le guerrier, avec l'bomo publice necans?4 $\mathrm{Si}$ l'historiographie française distingue trois dynamiques de changement historique, le sacrifice relèvera de la longue durée 5 . Selon l'école des Annales, la religion relève de la mentalité et donc de la longue durée, mais cette classification peut également servir à penser, autrement que par la perspective génético-biologique, les changements et les multiples différences du culte sacrificiel.

L'étroitesse et le caractère exclusif de cet accès au sacrifice apparaissent encore dans d'autres éléments réducteurs : le sacrifice est limité à l'animal dont on fait couler le sang, d'autres formes d'offrandes à la divinité sont par conséquent dissociées, comme la libation, le sacrifice préliminaire ${ }^{6}$, la fumigation d'encens, l'ex-voto, les offrandes d'argent, les fondations, les reliefs votifs ${ }^{7}$. Au système de l'économie des offrandes comme économie de la religion s'ajoutent des niveaux de description qui prennent en considération la forme culturelle du sacrifice.

En bref, ce que la science inclut dans la notion de "sacrifice», ou en exclut, doit être différencié dans sa diversité, ses fonctions multiples dans des temps différents, pour différentes divinités et rituels dans des endroits spécifiques. Le sacrifice a une histoire ${ }^{8}$.

${ }^{1}$ Les thèses d'Arnold Gehlen, exposées dans Der Mensch. Seine Natur und seine Stellung in der Welt, Berlin, Junker \& Dünnhaupt, 1940 et Urmensch und Spätkultur, Bonn, Athenäum, 1956, se trouvent à nouveau en partie chez Walter Burkert, comme, en quelque sorte, la thèse de l' «inondation par les stimuli»: le monde moderne attaque les hommes avec tant de stimuli simultanément qu'ils ne sont pas en mesure de les assimiler. Sur ce problème, voir Werner BREDE, « Mängelwesen », Historisches Wörterbuch der Philosopbie 5 (1980), p. 712 sq.

2 Walter BURKERT, Structure and History in Greek. Mythology and Ritual, Berkeley, CA, 1979 (Sather Lectures, 47). Walter Burkert, The Creation of the Sacred. Tracks of Biology in Early Religions (Gifford Lectures 1989), Cambridge, MA, Harvard, UP, 1996. Version all. : Kulte des Altertums. Biologische Grundlagen der Religion, München, Beck, 1998.

${ }^{3}$ Burkhard GladigOw, « Ovids Rechtfertigung der blutigen Opfer. Interpretationen zu Ovid. Fasti I 335-456 », Der Altsprachliche Unterricht 14.3 (1971), p. 5-23.

${ }^{4}$ Burkhard GLADIGOW, «Homo publice necans. Kulturelle Bedingungen kollektiven Tötens », Saeculum 37 (1986), p. 150-165.

${ }^{5}$ Burkhard GLADIGOW, "Elemente einer longue durée in der mediterranen Religionsgeschichte », in Ute PietruschKa (éd.), Gemeinsame kulturelle Codes in koexistierenden Religionsgemeinschaften. Leucorea-Colloquium Mai 2003, Halle, 2005 (Hallesche Beiträge zur Orientwissenschaft), p. 151-171.

${ }^{6}$ Petropoulou, Animal sacrifice, 2008, p. 38, n. 24 porte sur " the non-autonomous character of nonanimal sacrifice ». Eitrem a également appelé « sacrifices préliminaires » les cas où aucun sacrifice sanglant ne suivait; Sem. [Samuel] EITREM, Opferritus und Voropfer der Griechen und Römer Kristiania, 1915 (Videnskapsselskapets Skrifter: 2, Historisk-Filosofisk Klasse 1914, 1) [réimpression : Hildesheim, Olms, 1977).

7 Recherche lexicale du champ entier: Jean CASABONA, Recherches sur le vocabulaire des sacrifices en grec des origines à la fin de l'époque classique, Aix-en-Provence, Ophrys, 1966 (Université Aix-en-Provence. Publication des Annales de la Faculté des Lettres, 56). Christoph AufFarTH, "Für die Götter - für die Katz? Aspekte der Religionsökonomie im antiken Griechenland », in Hans G. KIPPENBERG, Brigitte LuCHESI (éd.), Lokale Religiongeschichte, Marbourg, 1995, p. 259-272.

8 « As regards to historical approaches to sacrifice [...] one could clearly state that historical theories on sacrifice are missing » : PETROPOULOU, Animal sacrifice, p. 27. 


\section{La fin du sacrifice : imposée ou expirée ?}

C'est alors qu'intervient la grande rupture : la longue durée se brise. On ne sacrifie plus. La fin du sacrifice est l'une des plus grandes ruptures dans l'histoire des religions. Par cette différence, l'histoire européenne des religions se démarque d'autres périodes de l'histoire des religions. La fin du sacrifice contribue puissamment à la façon dont les différentes religions se comprennent elles-mêmes.

Maria-Zoe Petropoulou traite ce point dans l'avant-propos de ce livre dont il est ici question $^{1}$ : éduquée dans le cadre de l'orthodoxie chrétienne en Grèce, elle a appris que le sacrifice est quelque chose que les Juifs de l'Ancien Testament ont accompli, mais que les Chrétiens ont rejeté. Précisément, le christianisme est né en tant que rejet du sacrifice sanglant. Mais ce n'est que plus tard qu'il lui est apparu clairement que les Grecs, à savoir "leurs ancêtres », ne construisaient pas uniquement des temples de marbre, mais y sacrifiaient également.

La vision idéologique de la rupture soutient que l'entrée du christianisme dans l'histoire des religions a entrainé la fin du sacrifice. D’abord comme refus, ensuite comme attaque polémique, ensuite comme jugulation violente, lorsque le christianisme est devenu religion d'État. Cette thèse a été renforcée par l'interprétation que ce ne sont pas les hommes qui ont instauré la violence, mais que Dieu lui-même est intervenu. Les Juifs auraient été punis pour la mise à mort du Christ par 1. la destruction de l'endroit où ils pouvaient sacrifier et donc 2. par l'obligation de faire ce que les Chrétiens faisaient par conviction : la fin du rite sacrificiel. En effet la destruction du temple de Jérusalem par les Romains a eu lieu tout juste 40 ans après la crucifixion, en l'an 70 ap. J.-C. Mais la juste punition pour la «mort de dieu » avait déjà été annoncée auparavant ${ }^{2}$.

Voilà pour l'idéologie relative à la fin du sacrifice dans le judaïsme. Mais comment l'aspect historique de la religion doit-il être évalué ? Comment se sont développés, d'un côté, la représentation et la pratique du sacrifice et, de l'autre, le rejet de celui-ci aussi bien à l'intérieur de la tradition religieuse elle-même que dans la pluralité des religions dans les cités antiques, où les Grecs, (les Romains), les Juifs et les Chrétiens habitaient si près l'un de l'autre et traitaient tant les uns avec les autres, que la religion était « inévitable » ? On rencontrait nécessairement la religion des autres. Les trois religions font toutes partie de la religion de la cité comme de la religion impériale romaine, c'est-à-dire qu'elles ne peuvent pas être considérées comme des religions distinctes dans un espace culturel ${ }^{3}$. Dans les trois traditions, on peut observer un développement, qui connaît tout d'abord, encore côte à côte, la pratique et la critique du culte. En d'autres termes, la critique du culte ne conduit pas à l'élimination de la pratique du sacrifice $^{4}$. Tout au plus mène-t-elle à des réformes ou à une meilleure justification. Mais il faut alors reconnaitre une lente transition du sacrifice animal comme rituel central de la religion vers une autre compréhension de la religion. La religion devient moins publique. Elle se

\footnotetext{
${ }^{1}$ Petropoulou, Animal Sacrifice, p. v-x.

${ }^{2}$ Les Chrétiens célèbrent une fête, un dimanche en été - pas très éloigné du jour d'anniversaire équivalent des Juifs le 9 av - où ils rappellent la destruction du second temple. Voir Christoph AufFARTH, "Christliche Festkultur und kulturelle Identität im Wandel: Der Judensonntag », in Benedikt KRANEMANN (éd.), Christliche Festkultur und die Mobilisierung von Religion in Europa, Münster, 2012, sous presse.

${ }^{3}$ Martin Hengel a diagnostiqué une hellénisation profonde pour le judaïsme en Palestine également: M. HeNGEL, Judentum und Hellenismus. Studien zu ibrer Begegnung unter besonderer Berücksichtigung Palästinas bis zur Mitte des 2. Jahrbunderts vor Christus, Tübingen, 1968.

${ }^{4}$ Burkhard GLADIGOW, «Opferkritik, Opferverbote und propagandistische Opfer », in Eftychia STAVRIAnopoulou, Axel Michaels, Claus Ambos (éd.), Transformations in Sacrificial Practices. From Antiquity to Modern Times, Münster, LIT, 2008 (Performanzen, 15), p. 263-287. Christoph AUfFARTH, «Euer Leib sei der Tempel des Herrns. Religiöse Sprache bei Paulus », in Dorothea Elm-VON DER Osten, Jörg RüPKE, Katharina WALDner (éd.), Texte als Medium und Reflexion von Religion im Römischen Reich, Stuttgart, 2006 (PawB, 14), p. 63-80.
} 
déplace dans la famille et la maison, dans des groupes et des lieux de réunion, dans des associations à mystères. L'acte sacrificiel est encore en partie pratiqué, mais il perd de plus en plus son sens de tous les jours. Et il est finalement abandonné dans toutes les traditions, par les Chrétiens, les Juifs et les cultes classiques. C'est ce point que Maria-Zoe Petropoulou traite dans son livre pour les différentes traditions religieuses, et non la thèse selon laquelle les Juifs et les païens auraient été finalement forcés à abandonner le sacrifice sous l'impulsion du modèle et l'emploi de la force des Chrétiens. Alors que l'empereur Julien, avec sa contrerévolution païenne, cherchait à réintroduire le sacrifice animal sanglant, même ses amis et partisans trouvaient cela horrible et répugnant ${ }^{1}$. Le temps du sacrifice était passé, en tout cas dans la religion de la cité. En d'autres termes, la fin du sacrifice provient des traditions religieuses elles-mêmes et n'est pas causée par une violence extérieure, d'une autre religion. En tout cas pas uniquement. Et inversement, la métaphore du sacrifice joue un rôle énorme, alors que le rite sacrificiel n'est plus pratiqué ${ }^{2}$, à peu près dans le sens de la mort de Jésus ${ }^{3}$. Une historisation des recherches sur le rite sacrificiel s'avère nécessaire. Les travaux ont déjà commencé : ils prennent en compte les différents médias, particulièrement les représentations iconographiques ${ }^{4}$, la littérature ${ }^{5}$, le droit ${ }^{6}$, l'histoire de la religion ${ }^{7}$.

Le livre de Maria-Zoe Petropoulou, Animal Sacrifice in ancient Greek religion, Judaism, and Christianity 100 BC - AD 200, est issu d'une thèse d'Oxford de 2004. Les sources sont citées en anglais dans l'argumentation (mais la consultation du texte grec en annexe est peu commode) et des informations de base, qui ne dépassent pas le niveau du dictionnaire, sont données de manière relativement introductive. C'est avec une méthode exemplaire que Petropoulou a évalué des sources littéraires du Ire siècle av. J.-C. jusqu'au II $^{\mathrm{e}}$ siècle ap. J.-C. et a également sélectionné des sources épigraphiques. Il faut cependant interroger l'intention des sources, seul moyen de comprendre leur contenu. À titre d'exemple, la critique de Pausanias sur la modification du rituel des enfants de Médée à Corinthe (Paus., II, 3, 7) se réfère au fait que, après 44 av. J.-C., des colons romains ont quitté l'Italie pour s'établir à Corinthe, ont fait revivre les cultes grecs, mais ont par la même occasion apporté des modifications arbitraires.

1 Voir la critique de l'un de ses partisans, l'historien Ammien Marcellin, Histoire romaine XXII, 12, 6. Nicole Belayche, "Sacrifice and Theory of Sacrifice During the Pagan Reaction. Julian the Emperor ", in Albert I. BAumgarTEN (éd.), Sacrifice in Religious Experience, Leiden, 2003 (SHR, 93), p. 101-126.

${ }^{2}$ Hildegard CANCIK-LINDEMAIER, «Opferphantasien. Zur imaginären Antike der Jahrhundertwende in Deutschland und Osterreich », in Der Altsprachliche Unterricht 14.3 (1987), p. 90-104. Repris dans Henriette HARICHSCHWARZBAUER; Barbara VON REIBNITZ (éd.), Von Atheismus bis Zensur. Römische Lektüren in kultumvissenschaftlicher Absicht, Würzburg, Königshausen, 2006, p. 193-210.

${ }^{3}$ Sur la mort du Christ comme « sacrifice » et les réserves sur cette interprétation, voir Henk S. VERSNEL, «Making Sense of Jesus' Death. The Pagan Contribution », in Jörg FrEY, Jens SCHRÖTER (éds.), Deutungen des Todes Jesu im Neuen Testament, Tübingen, Mohr Siebeck, 2005, p. 213-294. À l'inverse, Andreas BENDLIN, «Anstelle der anderen sterben: Zur Bedeutungsvielfalt eines Modells in der griechischen und römischen Religion », in J. Christine JANOWSKI, Bernd JANOwSKI, Hermann P. LiCHTENBERGER (éd.), Stellvertretung. Theologische, philosophische und kulturelle Kontexte, Vol. 1, Neukirchen/Vluyn, Neukirchener Verlag, 2006, p. 9-41.

${ }^{4}$ Nikolaus Himmelmann, Tieropfer in der griechischen Kunst, Opladen, 1997. Folkert T. VAN STRATEN, Hierà kalá. Images of animal sacrifice in archaic and classical Greece, Leiden, Brill, 1995 (RGRW, 127). Oliver HEKSTER et al. (éds.), Ritual Dynamics and Religious Change in the Roman Empire, Leiden, 2009 (Impact of Empire, 9) (y voir particulièrement Emily A. HEMELRIK, p. 253-267; Günther SCHÖRNER, p. 285-306).

${ }^{5}$ Dorothea Elm-von Der Osten, Jörg RÜPKE, Katharina WALDner (éd.), Texte als Medium und Reflexion von Religion im Römischen Reich, Stuttgart, 2006 (PawB, 14). Alessandro BARCHIESI, Jörg RÜPKE (éd.), Rituals in Ink, Stuttgart, Steiner, 2004 (PawB, 10).

${ }^{6}$ Clifford ANDO, Jörg RüPKE (éd.), Religion and law in classical and cbristian Rome, Stuttgart, Steiner, 2006 (PawB, 15).

${ }^{7}$ Jennifer Wright KnUST, Zsuzsanna VÁRHELYi (éd.), Ancient Mediterranean sacrifice, New York, Oxford UP, 2011. 
Dans d'autres cas, il ne formule pas cette critique ${ }^{1}$. Rien que sur Pausanias et son archaïsme, on a beaucoup travaillé ces dernières années et ce n'est guère pris en compte ${ }^{2}$. Lucien signale dans le De sacrificiis (12) un sacrifice qui n'est pas normal (Petropoulou, p. 40-42), mais s'exprime de manière cynique sur le comportement pré-culturel (" comme les Cyclopes ») des prêtres, qui se souillent de sang, bien qu'ils aient préalablement vérifié chaque recoin du lieu, pour vérifier si chacun avait les mains pures. On ne peut guère utiliser le texte pour invoquer la continuité ininterrompue du sacrifice, mais bien pour signaler le dégoût croissant face au sacrifice (et le fait que Lucien connait bien Homère) $)^{3}$. Les lectures étendues des sources juives du Ier siècle provenant de sources grecques (Philon et Josèphe) et rabbiniques, qui ont été attribuées à cette époque, sont donc dignes d'être mentionnées. Toutefois, les exigences en termes de compétence linguistique, la connaissance des contextes historiques et des dialogues cachés de l'intertextualité dépassent la capacité d'une seule chercheuse. Pour la métaphore du sacrifice chez les premiers Chrétiens, le problème est fondamental, parce que, sur le (règlement du) sacrifice juif, le rituel du bouc émissaire est pris comme référence (Lévitique 16, dans lequel le bouc émissaire n'est pas sacrifié; à la différence de l'agnus Dei dans Jesaja 53) ou parce que les Grecs et Romains récemment convertis cherchent une continuation de leur pratique sacrificielle dans les rites chrétiens. En résumé, la question est bonne, Petropoulou a donné une vue d'ensemble sur des sources pertinentes. Cependant, l'interprétation en est extrêmement difficile. Les impacts et les conséquences de la fin du sacrifice sont encore à rechercher. Cela ne peut se faire que dans le cadre d'un projet collectif qui rassemble des chercheurs de plusieurs horizons.

\section{Quelles conséquences la fin du sacrifice a-t-elle eu sur les religions antiques? Un projet de recherche sur la transformation de la religion antique}

Dans ses leçons au Collège de France (2004), Guy Stroumsa a décrit la transformation de la religion dans l'antiquité tardive en relation avec « la fin du rite sacrificiel »4. Il a mis en évidence, à juste titre, que la transformation du judaïsme n'est pas imposée de l'extérieur, mais représente une partie d'une réorientation interne du judaïsme. Cette réorientation commence déjà lors de la destruction du premier temple en 587 av. J.-C., mais le programme de la réforme cultuelle de Josias la précède d'une génération: si les sacrifices sont encore autorisés uniquement dans le temple de Jérusalem, la religion est déjà pour presque tous les Juifs une religion sans sacrifice sanglant - à l'exception du pèlerinage de Jérusalem ${ }^{5}$. L'alignement sur le commandement écrit du «Sinai » est proche, mais aussi en concurrence avec le temple de «Zion ». Stroumsa décrit la transformation - quelque chose d'unilatéral -

\footnotetext{
${ }^{1}$ Sur le patch-work gréco-romain du culte de Patras, voir Christoph AUFFARTH, «'Verräter - Übersetzer'? Pausanias, das römische Patrai und die Identität der Griechen in der Achaea ", in Hubert CANCIK, Jörg RüPKE (éd.), Reichsreligion und Provinzialreligion, Tübingen, Mohr, 1997, p. 219-238.

2 Dernièrement, l'ouvrage fondamental (paru trop tard pour Petropoulou) de Vinciane PIRENNEDelforge, Retour à la source. Pausanias et la religion grecque, Liège, Centre d'Étude de la Religion Grecque Antique, 2008 (Kernos, suppl. 20).

3 Sur ce point, Fritz GRAF, «Rejecting Sacrifice in Imperial Times: The Treatise On Sacrifice », dans Jennifer KNUST, Zsuzsanna VÁRHELYi (éd.), Ancient Mediterranean sacrifice, New York, Oxford, UP 2011, p. 203-213. N. BELAYCHE, «Entre deux éclats de rire. Sacrifice et représentation du divin dans le De sacrificiis de Lucien », in V. PirenNe-Delforge, F. Prescendi (éds.), Nourrir les dieux? Sacrifice et représentation du divin, Liège, 2011 (Kernos, suppl. 26), p. 165-180.

${ }^{4}$ Guy STRoumsa, La fin du sacrifice, Paris, 2005. - Traduction allemande Das Ende des Opferkultes, Berlin, Verlag der Weltreligionen, 2011. Mon compte rendu se trouve sur le site internet indiqué à la n. 39, p. 298.

${ }^{5}$ Beate EGO et al. (éd.), Gemeinde obne Tempel. Zur Substituierung und Transformation des Jerusalemer Tempels und seines Kults im Alten Testament, antiken Judentum und frühen Christentum, Tübingen, Mohr Siebeck, 1999. Y voir notamment Folker SIEGERT, «Die Synagoge und das Postulat des unblutigen Opfers », p. 335-356.
} 
avant tout comme une intellectualisation et une individualisation sur le modèle du rabbin. La transformation du judaïsme après le second temple est cependant beaucoup plus vaste. On doit en outre comprendre l'introduction du service à la synagogue et la transposition de la religion dans le cadre domestique (où la mère transmet la culture religieuse). Et cette transformation judaïque se trouve dans le contexte (chez Stroumsa: avant-garde) de la transformation des autres traditions religieuses dans la religion de l'empire romain : la $\lambda \circ \gamma \iota x \dot{\eta}$

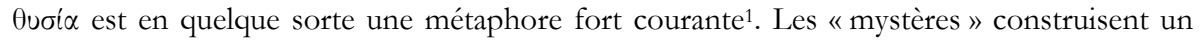
modèle pour la transformation ${ }^{2}$.

Les continuités doivent être mises en exergue au même titre que les caractéristiques nouvelles. Tout en gardant le même nom, qui assure une continuité, quelque chose de nouveau se développe. Ce qui concerne les « sacrifices mineurs » comme les sacrifices d'huiles dans les chandeliers, l'offrande de pain, l'offrande d'or, la fumigation d'encens, j'en ai présenté une vue d'ensemble : dans le christianisme, le sacrifice sanglant est strictement rejeté, mais les sacrifices mineurs restent la pratique rituelle 3 .

La tâche est complexe. Il s'agit de comprendre, d'une part, le processus de la fin du sacrifice comme un phénomène qui se développe à l'intérieur des religions, lié à la religion de l'antiquité tardive, et non comme une révolution de la fin du christianisme et une contrainte appliquée de l'extérieur. D'autre part, il faut aborder la transformation des lieux, pratiques et communautés religieux et, d'un point de vue linguistique, leur usage de concepts et de métaphores traditionnels, mais avec un nouveau contenu. Un tel projet ne peut être envisagé que par le biais d'une méthode interdisciplinaire et comparative. Ainsi, les négociations inter- et intrareligieuses de démarcation, de même que les similitudes, sont les plateformes fondamentales de la religion impériale antique dans le cadre de la transformation commune des traditions religieuses de l'antiquité tardive 4 .

Universität Bremen

Institut für Religionswissenschaft (Sportturm 6180)

Postfach 330440

D - 28334 BREMEM

Courriel:auffarth@uni-bremen.de

\footnotetext{
${ }^{1}$ Voir l'article fondamental de Hubert SEIWERT, « Opfer », Handbuch religionswissenschaftlicher Grundbegriffe 4 (1998), p. 268-84, ici p. 283. Les ouvrages sur le sacrifice en rapport avec le refus du sacrifice sanglant par les Chrétiens sont innombrables. Voici seulement quelques ouvrages récents: George HEYMAN, The Power of sacrifice. Roman and Christian discourses in conflict, Washington, DC, Catholic UP, 2007; Arnold ANGENENDT, Die Revolution des geistigen Opfers: Blut - Sündenbock - Eucharistie, Freiburg, Herder, 2011; Ann W. AstELl, Sandor GoOdHART (éds.), Sacrifice, Scripture, and Substitution. Readings in Ancient Judaism and Christianity, Washington, Notre Dame UP, 2011; Daniel C. Ullucci, The Christian Rejection of Animal Sacrifice, New York, Oxford UP, 2012; Bertram SCHMITZ, Vom Tempelkult zur Eucharistiefeier. Die Transformation eines Zentralsymbols aus religionswissenschaftlicher Sicht, Berlin, LIT, 2006.

${ }^{2}$ Christoph Auffarth, « Mysterienkulte », Reallexikon für Antike und Christentum (2012), sous presse.

${ }^{3}$ Christoph Auffarth, « Teure Ideologie - billige Praxis: Die ,kleinen' Opfer in der römischen Kaiserzeit ", in STAVRianOPOUlou et al. (éd.), Transformations in Sacrificial Practices, o.c. (p. 298, n. 23), p. 147-170.

${ }^{4}$ Voir mes comptes rendus de Daniel Boyarin, Borderlines (2004; trad. all. Abgrenzungen. Die Aufspaltung des Judäo-Christentums, 2009) et de Peter SCHÄFER, Die Geburt des Judentums aus dem Geist des Christentums, 2010, tous deux sur le site suivant http://buchempfehlungen.blogs.rpi-virtuell.net/.
} 